\title{
The Role of Nebulizer Gas Flow in Electrosonic Spray Ionization (ESSI)
}

\author{
Rui Wang, ${ }^{1}$ Pitt Allmendinger, ${ }^{1}$ Liang Zhu, ${ }^{1}$ Arto Juhani Gröhn, ${ }^{2}$ Karsten Wegner, ${ }^{2}$ \\ Vladimir Frankevich, ${ }^{1}$ Renato Zenobi ${ }^{1}$ \\ ${ }^{1}$ Department of Chemistry and Applied Biosciences, ETH Zurich, CH-8093 Zurich, Switzerland \\ ${ }^{2}$ Department of Mechanical and Process Engineering, ETH Zurich, CH-8092 Zurich, Switzerland
}

\begin{abstract}
In this work, we investigated the role of the nebulizer gas flow in electrosonic spray ionization (ESSI), by systematically studying the relation between the flow and the ion signals of proteins, such as cytochrome $c$ and holomyoglobin using ESSI-mass spectrometry (MS). When a neutral solution was delivered with a small sample flow rate $(\leq 5 \mu \mathrm{L} / \mathrm{min})$, no obvious transition from electrospray ionization (ESI) to ESSI was found as the gas velocity varies from subsonic to supersonic speed. Droplets mostly experienced acceleration instead of breakup by the highspeed nebulizer gas. On the contrary, using particular experimental conditions, such as an acidic solution or high sample flow rate $(\geq 200 \mu \mathrm{L} / \mathrm{min})$, more folded protein ions appear to be kept in droplets of diminishing size due to breakup by the high-speed nebulizer gas in ESSI compared with ESI. Theoretical analyses and numerical simulations were also performed to explain the observed phenomena. These systematic studies clarify the ionization mechanism of ESSI and provide valuable insight for optimizing ESSI and other popular pneumatically assisted electrospray ionization methods for future applications.
\end{abstract}

Key words: Electrosonic spray ionization, Electrospray ionization, Ionization mechanism

\section{Introduction}

耳 lectrosonic spray ionization (ESSI), first developed by Cooks' group in 2004 [1], is a combination of electrospray ionization (ESI) [2] and sonic spray ionization (SSI) $[3,4]$. Compared with the conventional ESI source, the pressure of the nebulizer gas in an ESSI source is much higher and can generate a supersonic flow, arguably rendering ESSI a very soft ionization technique. ESSI possesses more flexibility than conventional ESI: experimental parameters, such as sample flow rate, high voltage and geometry, can be changed over a wide range, providing more comprehensive control over the ionization process. This flexibility has proven valuable for different applica-

Correspondence to: Renato Zenobi; e-mail: zenobi@org.chem.ethz.ch tions. It has been reported that with the help of the supersonic gas flow, an ESSI source is able to keep the folded conformation of proteins and produce narrow peak widths and charge state distributions at lower charge states of multiply charged protein ions [1]. It has also been demonstrated that ESSI can be used as a powerful tool for investigating the noncovalent interaction between proteins and ligands due to its capability of preserving the noncovalent complex in the ionization process $[5,6]$.

The high-speed gas flow plays a central role in the ESSI technique. It not only lies at the origin of the versatility of ESSI but also brings additional complexity to the ionization process. However, there is hardly any literature studying how the gas flow influences the ionization process of analytes using ESSI, particularly with respect to the following questions: (1) Is there a clear switch-over from ESI to ESSI when the gas velocity is varied from subsonic to 
supersonic speed? (2) Is the preservation of the folded conformation of proteins in ESSI caused by the high-speed gas flow? (3) How does the nebulizer gas affect the droplet size and droplet velocity in the spray plume?

In the first paper on ESSI-MS [1], the authors compared the difference of the charge state distribution of protein ion peaks obtained by ESSI-MS with that produced by nano ESI-MS. However, other parameters also changed significantly in the comparison of these two ionization methods, e.g., the distance between the emitter of the capillary and the MS inlet, and the sample flow rate. To the best of our knowledge, there is still lack of a direct and comprehensive investigation on the relation between the charge state distribution of protein ion peaks and the nebulizer gas flow. While it is the gas velocity that is a key factor, the gas pressure is the parameter that is experimentally accessible. In this work, the gas pressure instead of gas velocity is thus reported when discussing the experimental results.

We systematically investigated the effect of the gas pressure on the MS signal of proteins. Two globular proteins, cytochrome $c$ and holomyoglobin, which bind the heme group differently, were studied. The heme group is covalently bound in cytochrome $c$, while it interacts noncovalently with myoglobin. This may cause different behavior upon changing the nebulizer gas flow. A comparison of the protein ion mass spectra, particularly of the charge state distribution as an important indicator for protein conformation changes [7-9], was done over a range of gas pressures with other parameters kept the same in the ESSI source. Experiments varying the solution $\mathrm{pH}$ and sample flow rates were performed to clarify the function of the nebulizer gas for keeping folded proteins during the ESSI process, especially at atypical experimental conditions. In addition, theoretical analyses and numerical simulations were conducted in this work to support the experimental findings.

\section{Experimental and Methods}

\section{Instrumentation}

A homebuilt ESSI source was used in this work as shown in Figure 1. The source was mounted on a XYZ stage in order to adjust the relative position between the source and the MS inlet. The ESSI source was perpendicular to the MS inlet, as in the typical "Z-spray" configuration. The vertical distance between the emitter of the capillary and the inlet was $10 \mathrm{~cm}$ and the horizontal distance was $0 \mathrm{~cm}$ (i.e., flush with the inlet cone), a standard geometry for ESSI sources $[1,6]$. All the capillaries of this source were made of untreated fused silica tubing (BGB Analytik, Böckten, Switzerland). The size of the inner capillary (for sample delivery) was $150 \mu \mathrm{m}$ o.d. and $50 \mu \mathrm{m}$ i.d., while that of the outer capillary was $350 \mu \mathrm{m}$ o.d. and $250 \mu \mathrm{m}$ i.d, the same as those used in previous work $[1,6]$. A high voltage power supply (HCN140-12500; FuG, Rosenheim, Germany) was used to

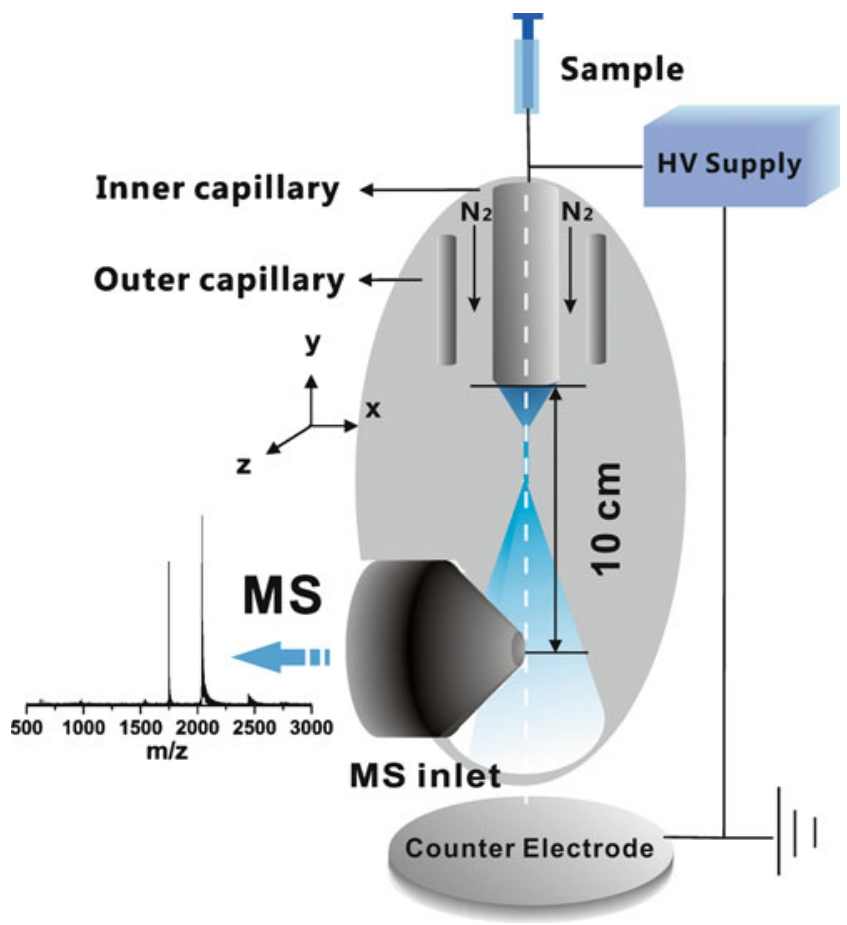

Figure 1. Schematic of the ESSI-MS setup

apply $4 \mathrm{kV}$ to the sample solution in all the experiments [6]. The sample solution was delivered by a $500 \mu \mathrm{L}$ or $5 \mathrm{~mL}$ syringe (Hamilton, Bonaduz, Switzerland) and a syringe pump (NE-1000; New Era Pump Systems, New York, NY, USA) resulting in flow rate of $5 \mu \mathrm{L} / \mathrm{min}$ or $200 \mu \mathrm{L} / \mathrm{min}$. Dry nitrogen was used as nebulizer gas, with a pressure varying from 5 to 45 bar, corresponding a gas volume flow rate of approximately 1.5 to $12 \mathrm{~L} / \mathrm{min}$ near the spray tip according to a calibration done previously [6].

ESSI mass spectra of cytochrome $\mathrm{c}$ and myoglobin were collected in the positive ion mode on a quadrupole time-offlight (Q-TOF) mass spectrometer (Q-TOF Ultima; Micromass/Waters, Manchester, UK). In the ESSI-MS measurements, the cone temperature was fixed at $50{ }^{\circ} \mathrm{C}$, and the cone and RF lens voltages were optimized for maximum ion signal. All mass spectra were accumulated for $80-100 \mathrm{~s}$ with $1 \mathrm{~s}$ scan time. The mass range was $\mathrm{m} / \mathrm{z} 500-3000$ in all measurements. The Mass Lynx software (v4.0; Waters, Manchester, U.K.) was used for analyzing the mass spectra. Complementary phase Doppler anemometry (PDA) measurements (TSI Inc., St. Paul, MN, USA) were done to investigate the droplet size and velocity in the spray plume.

\section{Sample Preparation}

Cytochrome $c$ from bovine heart (95\% HPCE; Fluka, Buchs, Switzerland; $\mathrm{MW}=12,327 \mathrm{Da}$ ) and holomyoglobin from horse heart ( $\geq 90 \%$; Sigma-Aldrich, Buchs, Switzerland; $\mathrm{MW}=17,568 \mathrm{Da}$ ) were used as model proteins in this work. In the experiments, they were dissolved in $100 \mathrm{mM}$ ammonium acetate solution at a concentration of $10 \mu \mathrm{M}$, 
with various $\mathrm{pH}$ values. The ammonium acetate solution was prepared from a $7.5 \mathrm{M}$ stock solution (SigmaFine Chemicals, Buchs, Switzerland) and water with a resistivity of 18 $\mathrm{M} \Omega \cdot \mathrm{cm}$ obtained from a NANO pure water purification system (Barnstead, IA, USA). The acidity of the solution was adjusted by adding acetic acid ( $>99.0 \%$; Fluka, Buchs, Switzerland).

\section{Numerical Simulations}

Full 3D numerical simulations were performed to calculate the gas flow pattern in the vicinity of the emitter of the sample capillary using a computational fluid dynamics (CFD) software package (ANSYS CFX 12.1; ANSYS, Berlin, Germany). The shear stress transport (SST) model was implemented in the simulation, with both laminar and turbulent flows considered. The inner side of the outer capillary and the outer side of the inner capillary were defined as no-slip walls. The dimensions of the inner and outer capillaries were the same as those in the ESSI-MS measurements. According to the experimental geometry of the ESSI source, the length of the outer capillary was fixed at $30 \mathrm{~mm}$ and the length of the inner capillary was $31 \mathrm{~mm}$. The inlets of the two capillaries were on the same plane, while the outlet of the inner capillary protruded $1 \mathrm{~mm}$ from the outlet of the outer capillary. The gas entered the inlet with a pressure varying from 5 to 45 bar. The outlet was set to open to the air (i.e., with 1 bar pressure). In order to simulate the distribution precisely, the mesh size was set to be as fine as $3 \mu \mathrm{m}$ around the emitter.

\section{Results and Discussion}

\section{Nebulizer Gas Flow Effect Using Neutral Solutions at a Sample Flow Rate of $5 \mu \mathrm{L} / \mathrm{min}$}

ESSI-MS measurements of cytochrome $c$ (cyt $c$ ) and holomyoglobin $(\mathrm{hMb})$ in ammonium acetate buffer at $\mathrm{pH}$ of 7.0 were first done, with various nebulizer gas pressures ranging from 5 to 45 bar. ESI-MS data without any nebulizer gas cannot be shown because no signals from protein ions were observed, presumably due to the fairly long distance between the emitter of the capillary and the MS inlet. As plotted in the inset of Figure 2, a narrow charge state distribution of cyt $c$ ions in the low charge state range $(6+$ to $7+)$ was observed, suggesting the generation of folded cyt $c$ ions [10]. However, there was no obvious shift in charge state with increasing gas pressure. Only the absolute intensity of the ion peaks increased clearly and the intensity ratio of the ion peak at charge state $7+$ to the peak at charge state $6+$ slightly rose as shown in Figure 2 . The improvement in absolute intensity can result from concentrating the plume and a better desolvation efficiency resulting from the high-speed gas flow. The surprising decrease of intensities at the highest gas pressure of 45 bar and the intensity ratio change of two peaks still need further study.

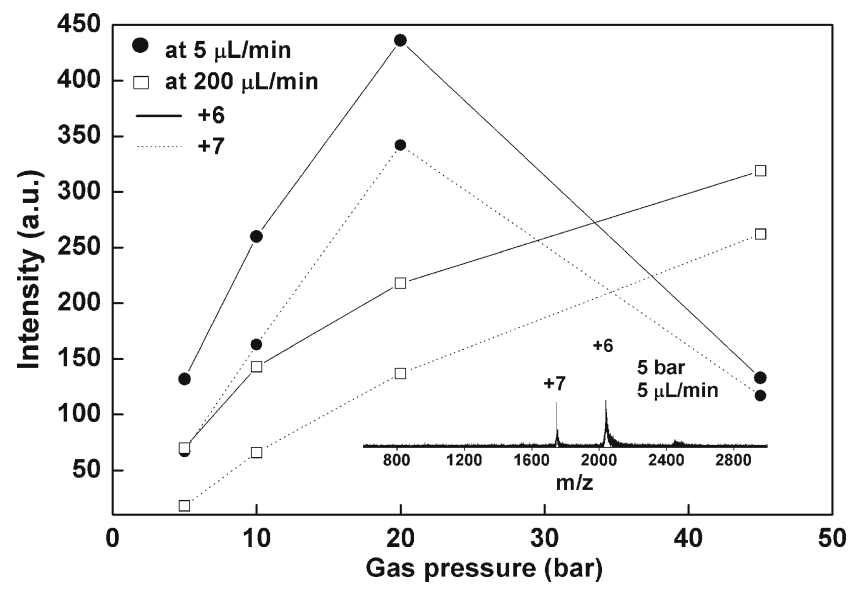

Figure 2. Intensity change as a function of gas pressure for charge states $6+$ and $7+$ for $10 \mu \mathrm{M}$ cytochrome $c$ in $100 \mathrm{mM}$ ammonium acetate buffer solution at various sample flow rates. The inset shows the mass spectrum of cytochrome $c$ with a sample flow rate of $5 \mu \mathrm{L} / \mathrm{min}$ at 5 bar nebulizer gas pressure

The results of $\mathrm{hMb}$ at a sample flow rate of $5 \mu \mathrm{L} / \mathrm{min}$ were similar to those of cyt $c$. A narrow charge state distribution of $\mathrm{hMb}$ ions in the low charge state range $(7+$ to $8+)$, but no distinct apomyoglobin $(\mathrm{aMb})$ ion peaks were observed at all pressures of the nebulizer gas. All these indicate the production of folded $\mathrm{hMb}$ ions during the ESSI process. There was no obvious shift in charge state with increasing gas pressure, either. Only the intensity ratio of charge state $8+$ to charge state $7+$ slightly increased when the gas pressure was raised, consistent with the results for cyt $c$. These results show that there is no switch-over of the charge state distribution of the proteins when the gas pressure of the nebulizer gas varied from 5 to 45 bar. In other words, the transition of the gas velocity from subsonic speed $(1.5 \mathrm{~L} / \mathrm{min})$ to supersonic speed $(12 \mathrm{~L} / \mathrm{min})$ probably has no substantial impact on the protein ions. A theoretical analysis in the subsequent section will support this observation.

\section{Nebulizer Gas Flow Effect Using Neutral Solutions at a Sample Flow Rate of $200 \mu \mathrm{L} / \mathrm{min}$}

Since no influence of the gas pressure on the ionization of the two proteins was observed at low sample flow rate, more ESSI-MS measurements of cyt $c$ and $\mathrm{hMb}$ were carried out at high sample flow rate to understand how the gas pressure change affects the protein ionization under particular conditions. A stable electrospray plume normally cannot be formed at very high sample flow rate. However, with the assistance of the high-speed nebulizer gas, the spray plume can be stabilized and the ion signals at high sample flow rate are comparable to or even better than the signals at low sample flow rate. Surprisingly, the mass spectra of cyt $c$ were very similar to these at small sample flow rate $(5 \mu \mathrm{L} /$ min). No distinct charge state shift was observed with 
increasing gas pressure. However, the change of the absolute intensity of the protein ion peaks was different between the two sample flow rates when gas pressure was varied as shown in Figure 2. At a nebulizer gas pressure of 5 bar, the intensity at high sample flow rate was lower than that at low sample flow rate, which might be insufficient to stabilize such high flow rate spray. However, when the gas pressure increased to $45 \mathrm{bar}$, the intensity at $200 \mu \mathrm{L} / \mathrm{min}$ was higher than that at $5 \mu \mathrm{L} / \mathrm{min}$. The high-speed nebulizer gas obviously increases the ion signal intensities at high sample flow rate, similar to those at low sample flow rate.

In contrast, $\mathrm{hMb}$ exhibits different behavior at high sample flow rate, as shown in Figure 3a. A broad charge state distribution of $\mathrm{aMb}$ ions in the range from $7+$ to $11+$ and a new peak of $\mathrm{hMb}$ at charge state $9+$ were observed. This indicates that $\mathrm{hMb}$ undergoes a conformational transition during the spray process. Larger droplets are produced at a sample flow rate of $200 \mu \mathrm{L} / \mathrm{min}$, which need a longer time to completely evaporate the solvent. The longer timescale of the evaporation enhances the probability that any change in the chemical environment inside the droplet, such as a decrease in $\mathrm{pH}$, induces a conformational change of the protein. Further studies will be done to clarify the conformational change in the neutral solution during the spray process. With increasing gas pressure, the intensities of the hMb ion peaks at charge state $7+$ and $8+$ increased, possibly due to a better desolvation efficiency of the folded hMb molecules, a temperature decrease caused by the adiabatic expansion of the nebulizer gas produced, or a reduced transit time of the droplets from the emitter to the entrance of the mass spectrometer.

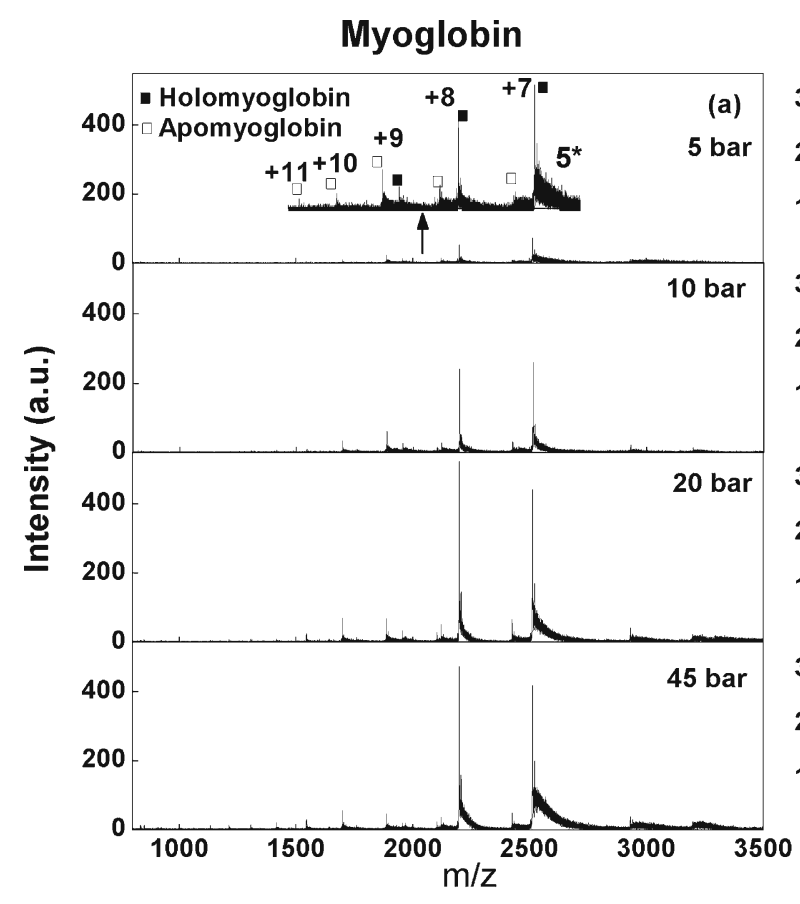

The distinct behavior of $\mathrm{hMb}$ and cyt $c$ was presumably on account of the different stabilities of these proteins. The unfolding free-energy changes in the absence of a denaturant $\left(\Delta G_{D}^{H_{2} O}\right)$ of $\mathrm{hMb}$ and cyt $c$ are 11.8 and $15.4 \mathrm{kcal} / \mathrm{mol}$ respectively [11], indicating that $\mathrm{hMb}$ is less stable than cyt $c$ in aqueous solution. Hence, compared with cyt $c, \mathrm{hMb}$ can be more easily disturbed by an environmental change occurring during the relatively long timescale of solvent evaporation.

\section{Nebulizer Gas flow Effect Using Acidic Solutions}

Dealing with proteins in acidic condition is also common in positive ion mode ESI measurements, since the presence of protons enhances the analyte ion signals. However, the conformation of proteins can easily change in acidic solution during the electrospray process [12-14]. Here, the influence of the gas pressure on the protein conformation in acidic solution was investigated. The mass spectra of cyt $c$ at a sample flow rate of $200 \mu \mathrm{L} / \mathrm{min}$ are shown in Figure $3 \mathrm{~b}$. Besides the folded cyt $c$ ion peaks at low charge state (6+ and $7+$ ), an additional distribution of ion peaks at higher charge states $(10+$ to $15+)$ was observed at 5 bar nebulizer gas pressure, indicating a transition of cyt $c$ from its folded form to an expanded structure, due to acid-induced unfolding $[7,15]$. With increasing gas pressure, the absolute intensity of the folded ion peaks obviously increased, i.e., the conformational transition of cyt $c$ was less pronounced with the assistance of the high-speed nebulizer gas. The reasons are likely exactly the same as those given above for

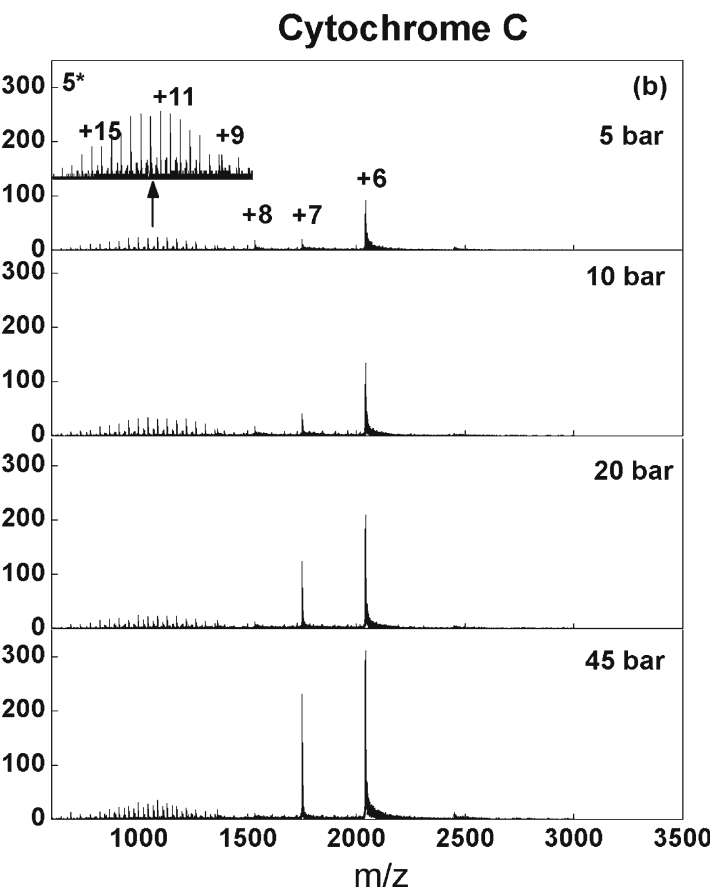

Figure 3. (a) Mass spectra of $10 \mu \mathrm{M}$ myoglobin in ammonium acetate buffer solution at various gas pressures (accumulation time was $80 \mathrm{~s})$. (b) Mass spectra of $10 \mu \mathrm{M}$ cytochrome $\mathrm{c}$ in acidic aqueous solution $(\mathrm{pH}=4.1)$ at various gas pressures (accumulation time was $100 \mathrm{~s}$ ). The sample flow rate was $200 \mu \mathrm{L} / \mathrm{min}$ 
$\mathrm{hMb}$. Similar phenomena were observed in ESSI-MS measurements of $\mathrm{hMb}$ (data not shown). All these results demonstrate that in acidic solution, more folded protein ions survive during the ESSI spray process with increasing gas pressure.

\section{Nebulizer Gas Flow Effect on the Electrosprayed Droplets}

\section{Numerical Simulations}

In order to better understand the behavior of the nebulizer gas flow around the emitter of the ESSI capillary, the gas velocity pattern close to the emitter was numerically simulated using the ANSYS software. In order to simplify the simulation, the droplet behavior was not accounted for. The gas velocity profiles at various gas pressures are shown in Figure 4. The simulation results are consistent with previous experimental observations. For example, Venter et al. measured the droplet velocity at $2 \mathrm{~mm}$ from the emitter of the capillary in a charged solvent spray with a high-speed nebulizer gas. It was found to be approximately $120 \mathrm{~m} / \mathrm{s}$ [16], suggesting the same value for the gas velocity due to the relatively short relaxation time of the droplets [17]. With a similar gas pressure implemented in our simulations, the gas velocity was found to be $108 \mathrm{~m} / \mathrm{s}$ at $2 \mathrm{~mm}$ from the emitter, very close to the estimated value of $120 \mathrm{~m} / \mathrm{s}$.

The contour profiles at pressure from 5 to 45 bar were very similar, only the absolute values of the gas velocity were different. The gas velocity decreased dramatically with distance from the emitter. For example, when a gas pressure of 5 bar was applied, the velocity close to the outlet of the outer capillary was $143 \mathrm{~m} / \mathrm{s}$, but dropped to only $2 \mathrm{~m} / \mathrm{s}$ $10 \mathrm{~mm}$ from the outlet of the inner capillary. With a gas pressure of $45 \mathrm{bar}$, the velocity was $600 \mathrm{~m} / \mathrm{s}$ at the outlet of the outer capillary and decreased to $16 \mathrm{~m} / \mathrm{s} 10 \mathrm{~mm}$ from the emitter. The average droplet velocity is around $10 \mathrm{~m} / \mathrm{s}$ in the stable electrospray plume without the nebulizer gas $[18,19]$.
When the gas velocity is close to or lower than the droplet velocity, the influence of the gas flow on the droplets is negligible (vide infra). This indicates that any significant interaction between the gas flow and the droplets must happen within $10 \mathrm{~mm}$ from the emitter.

The gas velocity profile at a pressure of 45 bar is shown as a zoomed view of the part, within $10 \mathrm{~mm}$ from the emitter, see Figure 5a. In order to compare this with the precise geometry of a stable electrospray plume, its position is superimposed on the graphic. The stable electrospray plume consists of a Taylor cone, a jet, and a plume. Based on previous experimental results and theoretical estimations $[20,21]$, the jet length is around $5 \mathrm{~mm}$ under the experimental conditions in this work. Thus, the spray plume should start $5 \mathrm{~mm}$ from the emitter, where the gas velocity is $66 \mathrm{~m} / \mathrm{s}$. A gas flow with this velocity cannot break up droplets smaller than $10 \mu \mathrm{m}$ (vide infra), which is the typical size in the stable spray plume [21].

In Figure 5b, the profile was enlarged further to show a zoomed view of the part $300 \mu \mathrm{m}$ from the emitter, to investigate exactly how the gas flow influences the Taylor cone and the jet part of the spray plume. A low gas flow region is formed in this area, due to the large gas pressure difference between the outlets of the inner and outer capillaries. Since conductive solutions were used as samples in this work, the Taylor cone can be taken to have a half angle of $49.3^{\circ}$, which is the value for a perfect conductor being sprayed [22]. Based on the inner diameter of the inner capillary and the angle, the length of the Taylor cone can be estimated to be $21.5 \mu \mathrm{m}$. The Taylor cone thus just penetrates into in the low gas flow region, where the velocity is between 6 and $90 \mathrm{~m} / \mathrm{s}$. The jet part is located in an area with a gas velocity ranging from 66 to $400 \mathrm{~m} / \mathrm{s}$. The jet diameter was estimated to be $6.3 \mu \mathrm{m}$ according to the experimental conditions [23]. This is consistent with a droplet size of 5-7 $\mu \mathrm{m}$ obtained by complementary phase Doppler anemometry (PDA) measurements of the ESI spray without nebulizer gas. With a high-speed nebulizer gas, the

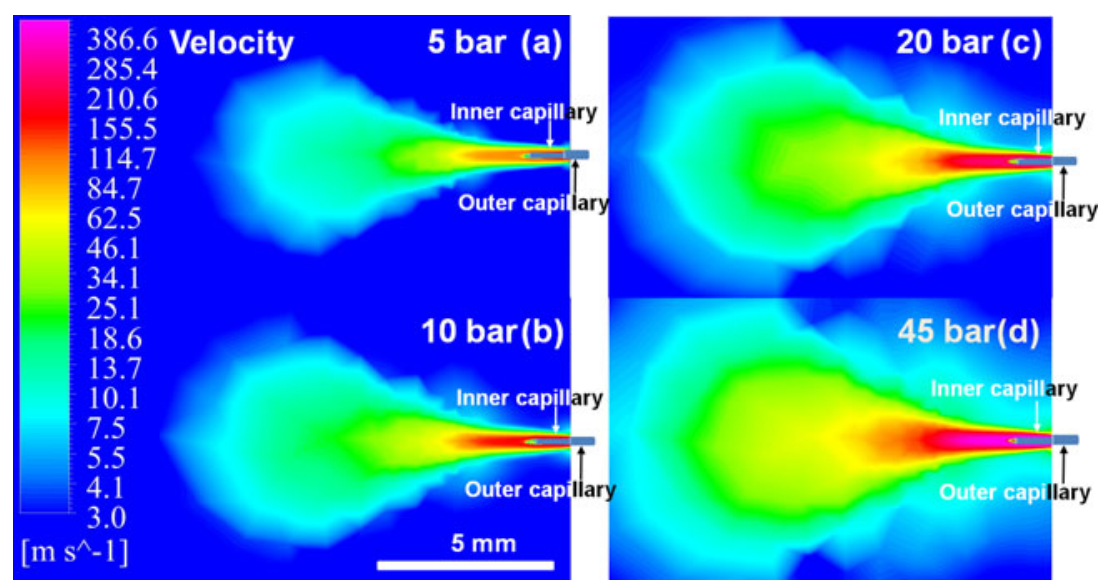

Figure 4. Simulation of gas velocity profiles at various gas pressures 


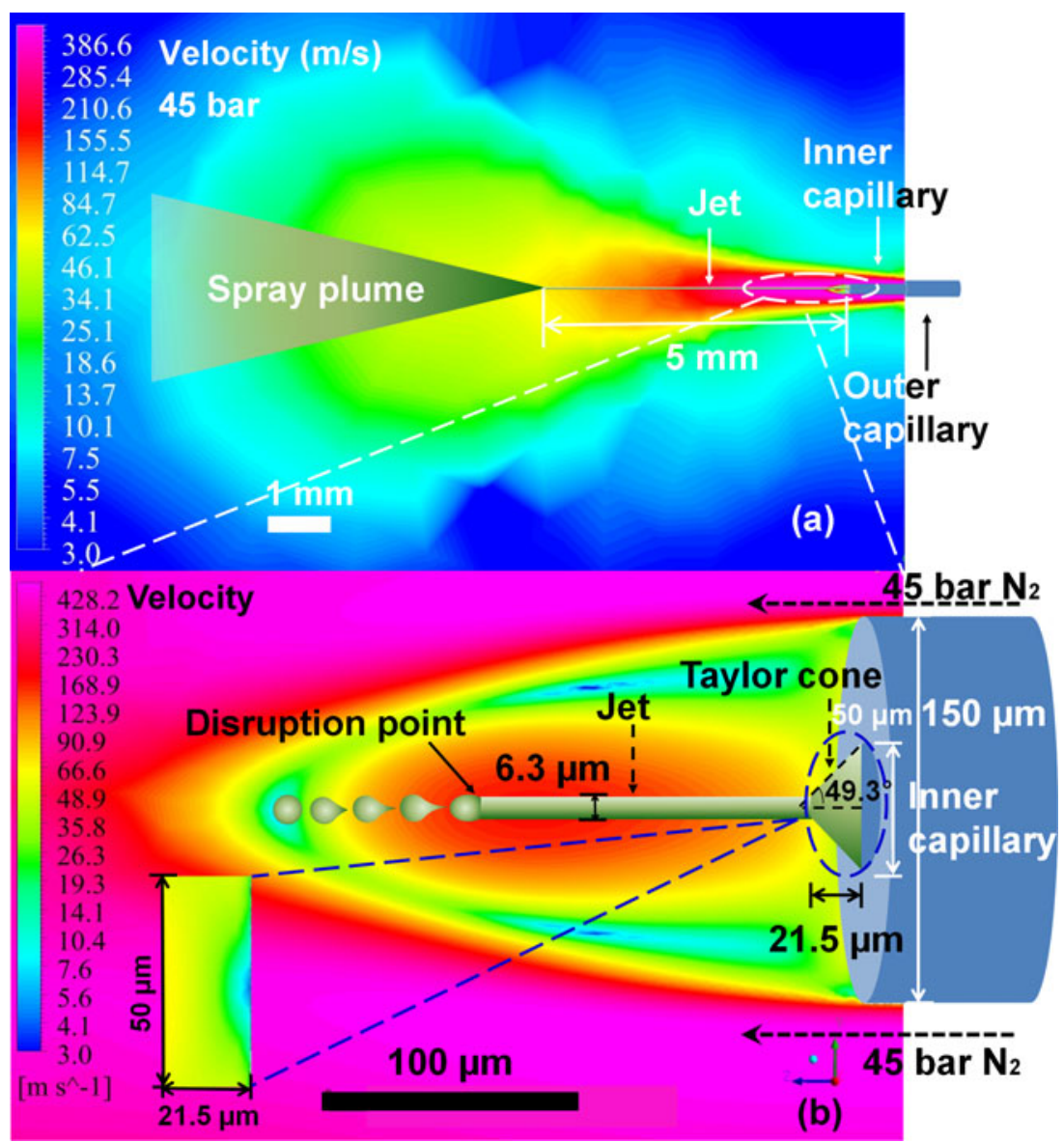

Figure 5. (a) Gas velocity map with a nebulizer gas of 45 bar pressure. A sketch of a stable electrospray plume is superimposed on the map to depict the geometry of the system. (b) Zoom-in view of the emitter part of panel (a) including the jet and Taylor cone of the spray plume. The inset of (b) shows the gas velocity distribution inside the Taylor cone area

disruption point of the jet, where first droplets appear, probably shifts closer to the emitter, due to the enhanced shear on the jet stream by the gas flow. However, the droplets at the disruption point of the jet, with a size close to the jet diameter of $6.3 \mu \mathrm{m}$, should mostly experience an acceleration instead of breakup by the gas flow as discussed in the following parts. Another key result of our PDA measurements is that the velocity of the droplets produced from the jet increased two times when a nebulizer gas of 5 bar pressure was used, but the size remained the same. More PDA measurements will be performed in the near future to clarify the influence of the nebulizer gas on the droplets.

The numerical simulation results discussed above demonstrate that the gas velocity has decreased dramatically $10 \mathrm{~mm}$ from the emitter, implying that the gas flow mostly influences the droplets within less than $10 \mathrm{~mm}$ from the emitter. A comparison of the geometry of a stable electrospray plume and the gas velocity profile provides useful information about the impact of the gas flow on the electrospray.

\section{Theoretical Analysis}

A theoretical analysis was also performed to explain the phenomena observed in the ESSI-MS experiments. Theoretically speaking, the nebulizer gas can influence the electrosprayed droplets during the spray process in three ways: (1) breakup of droplets; (2) acceleration of droplets; (3) acceleration of droplet desolvation.

\section{Breakup of Droplets}

When the ESSI droplets are suspended in a nebulizer gas flow with a much higher velocity, the gas flow exerts an aerodynamic force on the droplets. If the force is high enough, the droplets will break up. This process is also called "atomization" of droplets [15], a topic which has been thoroughly studied. A key parameter in this process is the Weber number, which is a dimensionless value used for analyzing complex fluidic flows with fluid-fluidic interfaces. It is known that droplets suddenly placed in a high-speed gas flow will experience breakup if the Weber number exceeds 
approximately 10 [24, 25]. The Weber number can be calculated as:

$$
W e=\frac{\rho_{g}\left(v_{g}-v_{d}\right)^{2} d}{\sigma}
$$

where $\rho_{g}$ and $v_{g}$ represent the density and velocity of the gas, $v_{d}$ and $d$ are the velocity and diameter of the droplet, and $\sigma$ is its surface tension. The minimum velocity of gas flow required for breakup can be estimated from Equation 1. For simplicity, only water and nitrogen are considered for the droplet and the gas, respectively. Without nebulizer gas, the average droplet diameter is approximately $5-10 \mu \mathrm{m}$ [21] and the average droplet velocity is $10 \mathrm{~m} / \mathrm{s}[18,19]$ for a stable electrospray at low sample flow rate. For a water droplet with $10 \mu \mathrm{m}$ diameter, the gas velocity relative to the droplet should be $240 \mathrm{~m} / \mathrm{s}$ to reach a Weber number of 10 . If the water droplet diameter decreases to $5 \mu \mathrm{m}$, the gas velocity relative to the droplet needs to be at least $339 \mathrm{~m} / \mathrm{s}$ to break up the droplet. When the sample flow rate is higher than $200 \mu \mathrm{L} / \mathrm{min}$, the initial droplet diameter can be as high as $50 \mu \mathrm{m}$ according to the size of the inner capillary. In this case, the gas velocity needs to be $107 \mathrm{~m} / \mathrm{s}$ for breakup. Furthermore, when the droplet size decreases, the droplet is easier to accelerate, resulting in the reduction of the relative velocity between the gas flow and droplets. Therefore, the acceleration of droplets by a high-speed gas flow also needs to be considered.

\section{Acceleration of Droplets}

When droplets are immersed in a high-speed gas flow, they will also be accelerated. The terminal velocity of a droplet depends on the relaxation time $\tau$, defined as [26]:

$$
\tau=\frac{\rho_{d} d^{2} C_{C}}{18 \eta}
$$

where $d$ and $\rho_{d}$ are the diameter and density of the droplet, $\eta$ is the coefficient of dynamic viscosity, and $C_{c}$ is the Cunningham correction factor. Equation 2 shows that a smaller droplet with less relaxation time will reach the gas velocity faster by the acceleration of the gas flow. Previous studies of the relaxation time show that a water droplet with a diameter of $50 \mu \mathrm{m}$ has a relaxation time of $8 \mathrm{~ms}$. When the size decreases to 10 and $1 \mu \mathrm{m}$, the relaxation times are $0.31 \mathrm{~ms}$ and $3.5 \mu \mathrm{s}$, respectively [26], Thus, a $4 \mu \mathrm{m}$ droplet with a relaxation time of $10 \mu \mathrm{s}$ [26] is small enough to closely follow the gas-phase flow almost immediately [27]. In other words, the droplet velocity can rapidly increase to the gas velocity when the droplet diameter is smaller than a few $\mu \mathrm{m}$. The Weber number will become very small in this case, due to the small value of $\left(v_{g}-v_{d}\right)$, and breakup will not take place according to Equation 1.

If the diameter of a droplet is larger than $50 \mu \mathrm{m}$, the relaxation time is too long to accelerate droplets and breakup of droplets driven by high sample flow rate should dominate.
This aerodynamic breakup should split droplets to a common size, which is relatively independent of the initial droplet size [28]. Zilch et al. suggested that after breakup, the final size of droplets converges to around $3 \mu \mathrm{m}$ [28]. Thus, it is difficult to have droplets much smaller than $3 \mu \mathrm{m}$ in the early part of an ESSI plume even with a supersonic nebulizer gas. This is consistent with previous results from Cooks' group [16]. They measured the droplet size in a charged solvent spray using a high-speed nebulizer gas $5 \mathrm{~mm}$ from the spray tip by a phase Doppler particle analyzer, and found the droplet diameter to vary from 2 to $6 \mu \mathrm{m}$, which is close to the final droplet size estimated theoretically.

\section{Acceleration of Droplet Desolvation}

When the nebulizer gas meets droplets in the spray, the gas flow can carry solvent vapor away from the surface of the droplet, thereby boosting evaporation. However, the nebulizer gas can also accelerate the droplets, reducing the time available to travel from the emitter of the capillary to the MS inlet, thus shortening the time available for solvent evaporation. These two factors have opposite effects on desolvation efficiency. Here, we discuss this issue using the evaporation rate equation $[26,29]$ :

$$
\begin{aligned}
N= & \frac{D\left(2.0+0.6\left(\rho_{d} d\left|v_{d}-v_{g}\right| / \mu\right)^{1 / 2}\left(\mu / \rho_{d} D\right)^{1 / 3}\right)}{d} \\
& \times\left(\frac{p_{\text {sat }}\left(T_{d}\right)}{R T_{d}}-X \frac{p_{\infty}}{R T_{\infty}}\right)
\end{aligned}
$$

where $R$ is the gas constant, $D$ is the diffusion coefficient of the vapor molecules, $d$ and $\rho_{d}$ represent the diameter and density of the droplet, $p_{\text {sat }}$ is the saturation vapor pressure at the temperature of the droplet $T_{d}$, and $p_{\infty}, T_{\infty}$, and $X$ are the pressure, temperature, and the mole fraction of the vapor well away from the droplet surface. $v_{d}$ and $v_{g}$ are the velocities of the droplet and nebulizer gas. The evaporation rate of the droplet thus depends on the relative velocity between the droplet and the nebulizer gas. When the droplet size is $\sim 4 \mu \mathrm{m}$, rapid acceleration of the droplet takes place and the droplet velocity increases to the gas speed, thereby decreasing the evaporation rate.

Thus, the desolvation efficiency for small droplets $(\leq 4 \mu \mathrm{m})$ cannot be dramatically improved by an increasing gas pressure in ESSI. This explains the phenomena observed in an ESSI-MS measurement of proteins in neutral solution at low sample flow rate. It also puts into question whether a new acronym (ESSI) is at all justified for ESI at elevated gas flows in this case. Droplets less than $10 \mu \mathrm{m}$ in diameter are produced at a $5 \mu \mathrm{L} / \mathrm{min}$ sample flow rate [21], i.e., very close to the $4 \mu \mathrm{m}$ size. These droplets mostly experience acceleration instead of breakup by the high-speed gas flow. As a consequence, there is no significant improvement of the desolvation efficiency by the high-speed nebulizer gas, meaning that the amount of water and ammonium acetate 
surrounding the proteins is not reduced rapidly. This cannot cause a significant conformational transition of the proteins. When the solution is acidic, acceleration of the small droplets can reduce the transit time from the emitter to the MS inlet, resulting in a lower probability for a conformational change, and the survival of more folded proteins during the ESSI process.

In the case of a high sample flow rate, initial droplets with diameters as large as $50 \mu \mathrm{m}$ or even more are formed. The breakup of droplets by the nebulizer gas dominates at the beginning of the ESSI plume. When the high-speed gas flow reduces the droplet size to $\sim 3 \mu \mathrm{m}$, which is similar to that in a stable electrospray at small sample flow rate, the acceleration becomes dominant. Thus, the mass spectra of the protein ions at a nebulizer gas pressure of 45 bar should be very similar even if very different sample flow rates are used, due to the similar final droplet size produced in the spray plume. This is exactly what was found experimentally.

\section{Conclusions}

In this work, measurements of cyt $c$ and $\mathrm{hMb}$ in neutral solution with various gas pressures show that there was no obvious change of the charge state distribution of protein ion peaks when the sample flow rate was $5 \mu \mathrm{L} / \mathrm{min}$, indicating that there was no switch-over of ionization from ESI to ESSI when the gas velocity varied from subsonic to supersonic speed. Droplets of small size produced by the stable electrospray mostly undergo acceleration instead of breakup by the high-speed nebulizer gas. However, under particular experimental conditions, such as low $\mathrm{pH}$ and high sample flow rate, the high-speed nebulizer gas helps proteins to keep their folded structures in the spray process. In acidic solution at small sample flow rates, the transit time of small droplets from the emitter to the MS inlet decreases because of the acceleration, resulting in a lower probability for unfolding of proteins. At high sample flow rates, much smaller droplets are produced due to breakup. The final droplet size in the plume is similar to that in the stable spray formed from neutral solutions at low sample flow rates.

\section{Acknowledgments}

The authors thank Dr. Elisabetta Boeri Erba and Mr. Konstantin Barylyuk for their kind help. This project was supported by the Swiss National Science Foundation (grant no. 200020-124663).

\section{References}

1. Takats, Z., Wiseman, J.M., Gologan, B., Cooks, R.G.: Electrosonic Spray Ionization. A Gentle Technique for Generating Folded Proteins and Protein Complexes in the Gas Phase and for Studying Ion-Molecule Reactions at Atmospheric Pressure. Anal. Chem. 76, 4050-4058 (2004)

2. Fenn, J.B., Mann, M., Meng, C.K., Wong, S.F., Whitehouse, C.M.: Electrospray Ionization for Mass-Spectrometry of Large Biomolecules. Science 246, 64-71 (1989)

3. Hirabayashi, A., Sakairi, M., Koizumi, H.: Sonic Spray Ionization Method for Atmospheric-Pressure Ionization Mass-Spectrometry. Anal. Chem. 66, 4557-4559 (1994)
4. Hirabayashi, A., Sakairi, M., Koizumi, H.: Sonic Spray MassSpectrometry. Anal. Chem. 67, 2878-2882 (1995)

5. Wiseman, J.M., Takats, Z., Gologan, B., Davisson, V.J., Cooks, R.G.: Direct Characterization of Enzyme-Substrate Complexes by Using Electrosonic Spray Ionization Mass Spectrometry. Angew. Chem. Int. Edit. 44, 913-916 (2005)

6. Jecklin, M.C., Touboul, D., Bovet, C., Wortmann, A., Zenobi, R.: Which Electrospray-Based Ionization Method Best Reflects ProteinLigand Interactions Found in Solution? A Comparison of ESI, NanoESI, and ESSI for the Determination of Dissociation Constants with Mass Spectrometry. J. Am. Soc. Mass Spectrom. 19, 332-343 (2008)

7. Chowdhury, S.K., Katta, V., Chait, B.T.: Probing Conformational Changes in Proteins by Mass-Spectrometry. J. Am. Chem. Soc. 112, 9012-9013 (1990)

8. Katta, V., Chait, B.T.: Observation of the Heme Globin Complex in Native Myoglobin by Electrospray-Ionization Mass-Spectrometry. J. Am. Chem. Soc. 113, 8534-8535 (1991)

9. Konermann, L., Collings, B.A., Douglas, D.J.: Cytochrome $c$ Folding Kinetics Studied by Time-Resolved Electrospray Ionization Mass Spectrometry. Biochemistry 36, 5554-5559 (1997)

10. Jarrold, M.F.: Unfolding, Refolding, and Hydration of Proteins in the Gas Phase. Acc. Chem. Res. 32, 360-367 (1999)

11. Pace, C.N.: The Stability of Globular Proteins. Crit. Rev. Biochem 3, 1$43(1975)$

12. Ideue, S., Sakamoto, K., Honma, K., Clemmer, D.E.: Conformational Change of Electrosprayed Cytochrome $c$ Studied by Laser-Induced Fluorescence. Chem. Phys. Lett. 337, 79-84 (2001)

13. Konermann, L., Rosell, F.I., Mauk, A.G., Douglas, D.J.: Acid-Induced Denaturation of Myoglobin Studied by Time-Resolved Electrospray Ionization Mass Spectrometry. Biochemistry 36, 6448-6454 (1997)

14. Konermann, L., Douglas, D.J.: Acid-Induced Unfolding of Cytochrome $c$ at Different Methanol Concentrations: Electrospray Ionization Mass Spectrometry Specifically Monitors Changes in the Tertiary Structure. Biochemistry 36, 12296-12302 (1997)

15. Goto, Y., Hagihara, Y., Hamada, D., Hoshino, M., Nishii, I.: AcidInduced Unfolding and Refolding Transitions of Cytochrome $c-a$ Three-State Mechanism in $\mathrm{H}_{2} \mathrm{O}$ and $\mathrm{D}_{2} \mathrm{O}$. Biochemistry 32, 1187811885 (1993)

16. Venter, A., Sojka, P.E., Cooks, R.G.: Droplet Dynamics and Ionization Mechanisms in Desorption Electrospray Ionization Mass Spectrometry. Anal. Chem. 78, 8549-8555 (2006)

17. Heine, M.C., Pratsinis, S.E.: Droplet and Particle Dynamics During Flame Spray Synthesis of Nanoparticles. Ind. Eng. Chem. Res. 44, 6222-6232 (2005)

18. Tang, K., Gomez, A.: On the Structure of an Electrostatic Spray of Monodisperse Droplets. Phys. Fluids 6, 2317-2332 (1994)

19. Olumee, Z., Callahan, J.H., Vertes, A.: Droplet Dynamics Changes in Electrostatic Sprays of Methanol-Water Mixtures. J. Phys. Chem. A 102, 9154-9160 (1998)

20. Gamero-Castano, M.: Characterization of the Electrosprays of 1-Ethyl3-Methylimidazolium bis(Trifluoromethylsulfonyl) Imide in Vacuum. Phys. Fluids 20, 11 (2008)

21. Wortmann, A., Kistler-Momotova, A., Zenobi, R., Heine, M.C., Wilhelm, O., Pratsinis, S.E.: Shrinking Droplets in Electrospray Ionization and Their Influence on Chemical Equilibria. J. Am. Soc. Mass Spectrom. 18, 385-393 (2007)

22. Taylor, G.: Disintegration of Water Drops in Electric Field. Proc. $R$. Soc. Lond. A Math. Phys. Sci. 280, 383-397 (1964)

23. Cloupeau, M., Prunetfoch, B.: Electrohydrodynamic Spraying Functioning Modes: A Critical Review. J. Aerosol Sci. 25, 1021-1036 (1994)

24. Wierzba, A.: Deformation and Breakup of Liquid-Drops in a GasStream at Nearly Critical Weber Numbers. Exp. Fluids 9, 59-64 (1990)

25. Krzeczkowski, S.A.: Measurement of Liquid Droplet Disintegration Mechanisms. Int. J. Multiph. Flow 6, 227-239 (1980)

26. Hinds, W. C. Aerosol Technology, 2nd ed.; John Wiley and Sons, Inc.: New York, 1999.

27. Hardalupas, Y., Liu, C.H., Whitelaw, J.H.: Experiments with Disk Stabilized Kerosene-Fueled Flames. Combust. Sci. Technol. 97, 157191 (1994)

28. Zilch, L.W., Maze, J.T., Smith, J.W., Ewing, G.E., Jarrold, M.F.: Charge Separation in the Aerodynamic Breakup of Micrometer-Sized Water Droplets. J. Phys. Chem. A 112, 13352-13363 (2008)

29. Wilhelm, O., Madler, L., Pratsinis, S.E.: Electrospray Evaporation and Deposition. J. Aerosol Sci. 34, 815-836 (2003) 